\title{
Evolution of BDNF Full-Length/Truncated Receptor Ratio and Cognitive/General Functioning After a First Episode of Psychosis
}

Mónica Martínez-Cengotitabengoa ${ }^{1,2,3,4,4}$, Susana Alberich ${ }^{1,2}$, Mara Parellada ${ }^{2,5}$, Bibiana Cabrera ${ }^{2,6}$, Esther Berrocoso 2,7, Roberto Rodriguez ${ }^{2,8}$, Antonio Lobo ${ }^{2,9}$, María Paz García-Portilla²,10, Miquel Bernardo ${ }^{2,11}$, Juan Carlos Leza ${ }^{2,12}$, Ana González-Pinto ${ }^{1,2,3}$, FlammPEPs study*.

\begin{abstract}
Brain plasticity has demonstrated to play a role in the pathophysiology of schizophrenia. Cognitive deterioration in these patients can be prevented by ensuring the adequate functioning of signaling pathways associated with brain plasticity. As BDNF exerts its action through receptors, in this study, we hypothesized that levels of some BDNF receptors during a first episode of psychosis (FEP) would correlate with the cognitive and global functioning of patients in the long term. We also hypothesized that the improvement of the ratio of full-length (TrKB-FL) and truncated (TrKB-T) TrKB receptors, and the predominance of the full-length isoform would be associated with better cognition and functioning.
\end{abstract}

Peripheral levels of full-length (TrKB-FL) and truncated (TrKB-T) TrKB receptors were assessed in a sample of 97 FEP patients and 97 matched healthy controls. TrKB-FL/TrKB-T ratio

\footnotetext{
'Biomedical Research Networking Centre in Mental Health (CIBERSAM), Madrid, Spain

${ }^{2}$ Araba University Hospital, BioAraba Health Research Institute, Vitoria, Spain

${ }^{3}$ University of the Basque Country (UPV/EHU)

${ }^{4}$ National Distance Education University (UNED), Vitoria, Spain

${ }^{5}$ Child and Adolescent Psychiatry Department, Hospital General Universitario Gregorio Marañón School of Medicine, Universidad Complutense, liSGM, Madrid, Spain

${ }^{6}$ Department of Psychiatry and Clinical Psychobiology, University of Barcelona, Institut d'Investigacions Biomèdiques August Pi i Sunyer (IDIBAPS), Barcelona, Spain

${ }^{7}$ Area of Psychobiology. Department of Psychology. Neuropsychopharmacology \& Psychobiology Research Group, University of Cadiz, Cadiz, Spain Instituto de Investigación e Innovación en Ciencias Biomédicas de Cádiz, INiBICA, Hospital Universitario Puerta del Mar, Cádiz, Spain

${ }^{8}$ Research Institute of Hospital 12 de Octubre (i+12), Madrid, Spain

${ }^{9}$ Department of Psychiatry, Instituto de Investigación Sanitaria Aragón and University of Zaragoza, Spain

${ }^{10}$ Department of Psychiatry, Faculty of Medicine, University of Oviedo Instituto Universitario de Neurociencias del Principado de Asturias (INEUROPA), Oviedo, Spain

"Barcelona Clínic Schizophrenia Unit, Neuroscience Institute, Hospital Clínic de Barcelona, Spain

${ }^{12}$ Department of Pharmacology, Faculty of Medicine, Universidad Complutense de Madrid, Research Institute of Hospital 12 de Octubre (i+12) \& IUINQ Madrid, Spain

${ }^{\dagger}$ Author for correspondences: Mónica Martínez-Cengotitabengoa, Department of Psychiatry, Araba University Hospital, Olaguibel Street 29, 01004, Vitoria, Spain; tel: + 34 945007764, fax: + 34 945007764, e-mail: monica.martinezcengotitabengoa@osakidetza.eus

*Flamm-PEPs is a multicentre, collaborative, translational research study conducted by a CIBERSAM group. This study investigates inflammatory pathways in psychosis, both as potential biomarkers and as potential new therapeutic targets. Flamm-PEPs forms part of the PEPs study, a Spanish research project on first-episode psychosis.
} 
(hereinafter, FL/T) was calculated for each patient. Cognitive and global functioning was measured at inclusion and at two years.

A high baseline $\mathrm{FL} / \mathrm{T}$ ratio was found to be related to a better cognitive function (global cognition, verbal memory, working memory and premorbid IQ). Cognitive performance at disease onset and at two years improved when the levels of the ratio were higher than one, with functional BDNF receptor (TrKB-FL) exceeding the value of the truncated isoform (TrKB-T).

In addition the increase in the $\mathrm{FL} / \mathrm{T}$ ratio during the two years of follow-up had positive effects on global functioning. This may be due either to a reduction in TrKB-T or to an increase in TrKB-FL, or both. In conclusion FL / T ratio was related to general functioning and cognition in the long-term.

\section{Keywords}

Pathophysiology, Schizophrenia, Cognitive deterioration

\begin{abstract}
Introduction
Schizophrenia has multiple etiologies. In most cases, its physiopathology is related to neurodevelopmental disorders [1]. Patients generally show some degree of cognitive impairment before and after the onset of the disease. One of the main hypotheses about the etiology of schizophrenia considers that it is related to alterations in brain plasticity [2]. Brain plasticity is provided by neurotrophic agents, being the Brain Derived Neurotrophic Factor (BDNF) the most widely studied agent in patients with psychosis [2-4].
\end{abstract}

The cognitive function of patients with psychosis is impaired due to neurotrophic repair system dysfunction [5]. A recent study revealed that patients' functioning and response to antipsychotic therapy are directly related to appropriate BDNF signaling [6]. In addition some meta-analyses demonstrate the role of BDNF in schizophrenia [3,7]. Conversely, its role in cognition is more controversial. While some studies report an association of BDNF with planning ability [8], reasoning and problem solving [9], no association was found in other studies [10]. BDNF binds, among others, to two types of cell surface receptors: a totally functional form (TrKB-FL) and a truncated nonfunctional form (TrkB-T) that impairs the repair function of BDNF by competing for binding to TrKBFL [11]. These receptors have been shown to play a crucial role in cognitive performance in rat models of schizophrenia [12] and Alzheimer's disease[13]. Specifically, when BDNF/TrkB agonist was given to rats, their cognitive impairment improved. Nevertheless, to our knowledge, no studies have been performed to assess the relationship between BDNF receptors and cognitive function in patients with psychosis. Thus, we hypothesized that the levels of some BDNF receptors during a first episode of psychosis (FEP) would correlate with the cognitive and global functioning of patients in the long term. We also hypothesized that the improvement of the ratio of full-length (TrKBFL) and truncated (TrKB-T) TrKB receptors and predominance of the full-length isoform would be associated with better cognition and functioning.

The objective of this study was to determine whether the expression and evolution of some BDNF receptors are associated with the cognitive and global functioning of FEP patients in the long term ( 2 years). We also hypothesized that the improvement of the ratio of fulllength (TrKB-FL) and truncated (TrKB-T) TrKB receptors and predominance of the fulllength isoform would be associated with better cognition and functioning

\section{Material and Methods}

This study was performed within the framework of the longitudinal multicenter Flamm-PEPs study, which protocol has been described elsewhere [14]. The Flamm-PEPs study included 117 patients (aged between 9-35 years) with a FEP, and 106 age- and sex-matched healthy controls. The inclusion criteria for the group of patients were: 1) positive psychotic symptoms for less than 12 months, 2) Age of 9-35 at disease onset, 3) speaking Spanish fluently, and 4) giving written informed consent. Psychotic symptoms were assessed by the Positive and Negative Symptoms Scale (PANSS)[15]. Exclusion criteria for patients were: 1) mental retardation according to DSM-IV criteria, 2) history of 
head trauma with loss of consciousness, and 3) systemic disease with mental repercussions. Inclusion criteria for healthy controls were: 1) no past or current psychiatric disorder (DSMIV criteria), 2) speaking fluent Spanish, and 3) giving written informed consent. Exclusion criteria for healthy controls were the same as for patients plus a history of psychotic episodes in first-degree relatives. No participants had fever or any allergies, ongoing infections, or other serious physical conditions. No participants had received immunosuppressive drugs or vaccines in the last six months or anti-inflammatory drugs at least two days before blood sampling. In this sub study, we only included the 97 patients for whom baseline blood samples were available and who underwent neuropsychological assessment at baseline and at two years of follow-up. We also included 89 healthy controls, 55 of whom completed follow-up. As the sample of interest was the group of patients, the evolution of controls was not analyzed and their data were not included in longitudinal analyses. The study protocol was approved by the Ethics Committees of the six participating hospitals. Informed consent was obtained from all participants.

\section{- Data Collection}

After overnight fasting, venous blood was collected between 8 a.m. and 10 a.m. Blood collection and processing methods used in the Flamm-PEPs study are detailed at www.cibersam.es and have been described elsewhere[14]. Briefly, blood tubes were centrifuged $\left(641 \mathrm{~g} \mathrm{x} 10 \mathrm{~min}, 4^{\circ} \mathrm{C}\right)$ for plasma extraction. Plasma was stored at $-80^{\circ} \mathrm{C}$. The remaining sample was diluted 1:2 in culture medium (Roswell Park Memorial Institute RPMI 1640, Invitrogen). A gradient with Ficoll-Paque was used to isolate mononuclear cells by centrifugation $(800 \mathrm{~g} \times 40 \mathrm{~min}$, room temperature). PBMC layer was aspired and resuspended in RPMI and centrifuged $(1116 \mathrm{~g}$ $\times 10 \mathrm{~min}$, room temperature). The supernatant was removed and the mononuclear cell-enriched pellet was manually resuspended in RPMI and stored at $-80^{\circ} \mathrm{C}$.

BDNF expression levels in plasma were determined by enzymatic testing (Ray Biotech). Plasma BDNF levels reflect BDNF expression in the brain both in animal models and humans [16,17]. Full-length (TrKB-FL) and truncated (TrKB-T) receptor expression was determined in peripheral blood mononuclear cells (PBMCs) by Western-Blot analysis. The relative expression of the two receptors was calculated as the TrKBFL/TrkB-T ratio (hereinafter, $\mathrm{FL} / \mathrm{T}$ ratio). Data of the FL/T ratio in this sample have been published previously [6].

Cognitive function was assessed in patients at baseline and at two years after disease onset by evaluating the following domains: speed of processing, attention, verbal memory, working memory, executive function and estimated premorbid intelligence coefficient (Figure 1). Cognitive Global Index (CGI) was calculated by averaging the scores on cognitive domains, except for estimated premorbid IQ, which was used as an adjustment variable in statistical analyses. Tests were administered by experienced psychologists by order of difficulty from least to most difficult to reduce the effect of fatigue and facilitate participant's cooperation. Inter-rater reliability between two of the tests proved to be good to excellent $($ ICC >0.80). The two tests included the WAIS vocabulary subtest and the WCST, in which the final score may partially depend on the judgement of the rater. Finally, global functioning was evaluated using the GAF scale [18].

\section{- Statistical analysis}

Multiple linear regressions were used to analyze the relationship between BDNF receptor expression, cognitive performance and global functioning. Regressions were adjusted for potential confounding variables such as gender, age, civil status, education, socioeconomic level, body mass index, antipsychotic treatment, diagnosis, tobacco, alcohol or other drugs. The final model was selected by a backwards stepwise procedure. Only the variables that caused a significant change in the regression coefficient for the independent variable or those found to be significant were maintained in the model. Finally, interactions were determined. Once the final model had been constructed, adjustment and normality of residuals were tested to determine the reliability of the model. In the case of the FL/T ratio, we used the quantitative variable for preliminary analysis and its qualitative form in order to explore the influence of the proportion of each receptor, taking 1 as threshold value. Regression data are expressed as $\beta$-coefficients and $p$-values. In the presence of significant interactions, stratified effect size (95\% CI) on the dependent variable was calculated for different values of the independent variable $\left(3^{\text {rd }}, 25^{\text {th }}, 50^{\text {th }}\right.$, $75^{\text {th }}$ and $97^{\text {th }}$ percentiles). 


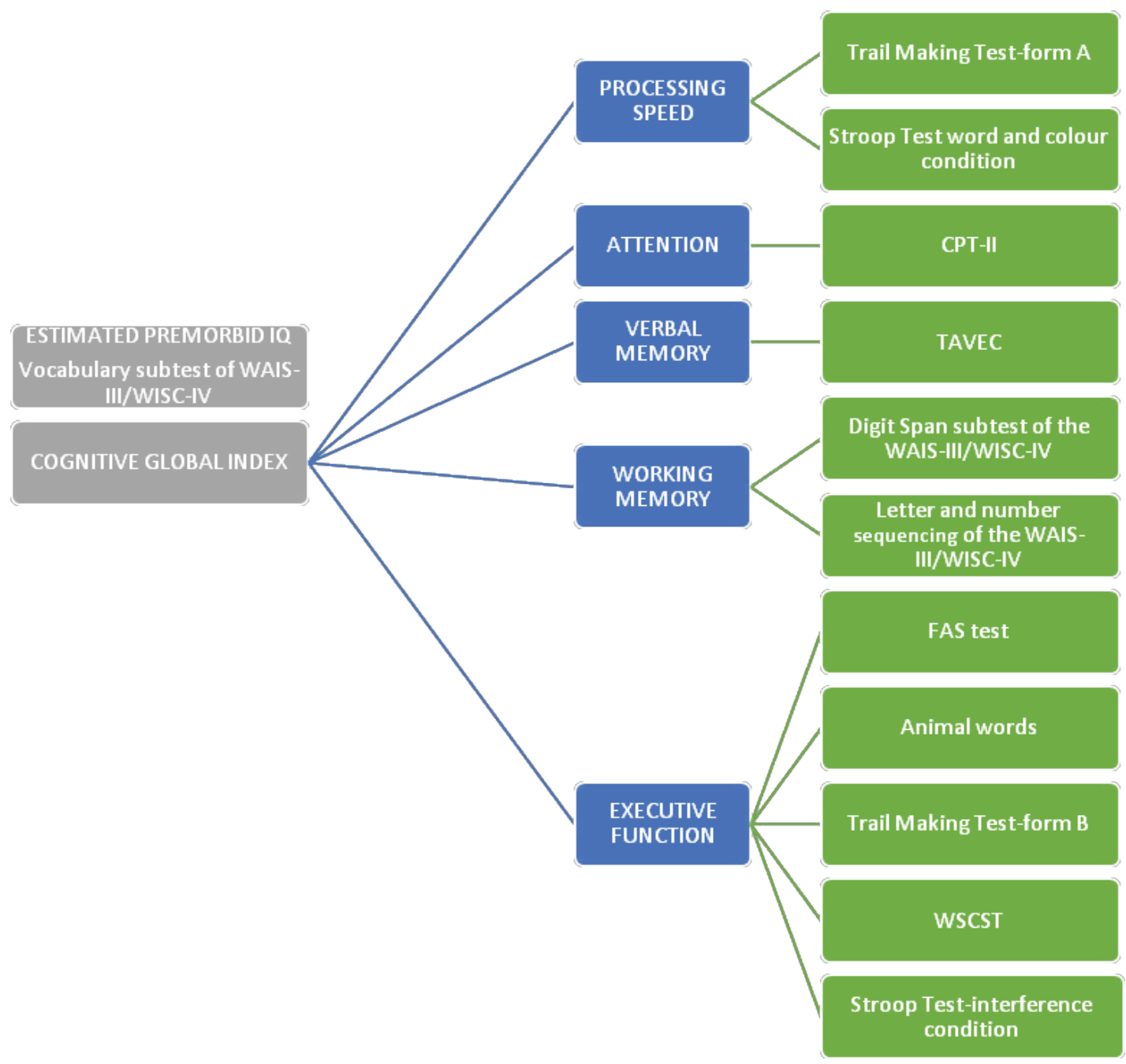

Figure 1: Tests included in each cognitive domain.

The association between shifts in BDNF receptor expression and cognitive and functional performance at two years was assessed by repeated measures ANOVA.

Changes in cognitive domains from baseline to 2 years were evaluated by Student's $t$-test for repeated measures.

Statistical analysis was performed using the Stata 12.1 software package. A $p<0.05$ was considered statistically significant.

\section{Results}

Table 1 summarizes scores on the different cognitive domains evaluated both at baseline and at two years of follow-up. Data are presented as means and standard deviations. All values are $\mathrm{Z}$ scores except for premorbid IQs, which are presented as scalar scores.
In this sample of patients, plasma BDNF levels were not significantly related to cognitive performance. A high baseline FL/T ratio correlated with a better baseline cognitive function, as assessed by the cognitive global index $(\beta=20.916, p=0.015)$ in FEP patients. No associations were observed in healthy controls $(\beta=3.58, \mathrm{p}=0.278)$.

The patients who experienced an increase in the FL/T ratio six months after FEP showed a better global functioning in the long term (as assessed by the GAF scale), as compared to patients who experienced a decrease in the $\mathrm{FL} / \mathrm{T}$ ratio $(\mathrm{F}=$ 213.90, $p<0.001$ ) (Figure 2).

As to specific cognitive domains, verbal memory was also related to a higher FL/T ratio $(\beta=0.885$, $\mathrm{p}=0.018$ ). Interaction between FL/T ratio and educational level was significantly related to baseline IQ and working memory $(\beta=-36.047$, $p=0.003$ and $\beta=-1.523, p=0.036$ respectively). 


\begin{tabular}{|c|c|c|c|c|c|c|}
\hline & \multicolumn{3}{|l|}{ Patients } & \multicolumn{3}{|l|}{ Controls } \\
\hline & Baseline & Two-year follow-up & Statistic & Baseline & Two-year follow-up & Statistic \\
\hline Premorbid IQ & $95.54(14.63)$ & $98.39(14.43)$ & $t=-2.029, p=0.047$ & $108.14(11.23)$ & $111.00(11.11)$ & $t=-1.123, p=0.266$ \\
\hline Processing speed & $-0.74(0.93)$ & $-0.45(0.87)$ & $t=-3.129, p=0.003$ & $0.31(0.76)$ & $0.39(0.66)$ & $t=-0.432, p=0.667$ \\
\hline Attention & $0.50(0.73)$ & $0.16(0.84)$ & $t=3.423, p=0.001$ & $-0.09(0.83)$ & $-0.17(0.75)$ & $t=-0.265, p=0.792$ \\
\hline Verbal memory & $-0.95(1.14)$ & $-0.43(0.99)$ & $t=-3.994, \mathrm{p}<0.001$ & $0.05(0.72)$ & $0.42(0.54)$ & $t=-4.342, \mathrm{p}<0.001$ \\
\hline Working memory & $-0.63(0.72)$ & $-0.54(0.87)$ & $t=-1.144, p=0.257$ & $0.08(0.87)$ & $0.20(0.91)$ & $t=-0.817, p=0.417$ \\
\hline Executive function & $-0.43(0.51)$ & $-0.24(0.76)$ & $t=-1.055, \mathrm{p}=0.314$ & $0.03(0.28)$ & $0.13(0.34)$ & $t=-0.483, p=0.637$ \\
\hline Cognitive global index & $-2.21(2.61)$ & $-1.38(2.65)$ & $t=-0.818, p=0.431$ & $0.19(0.37)$ & $0.25(0.26)$ & $t=-1.285, \mathrm{p}=0.221$ \\
\hline
\end{tabular}

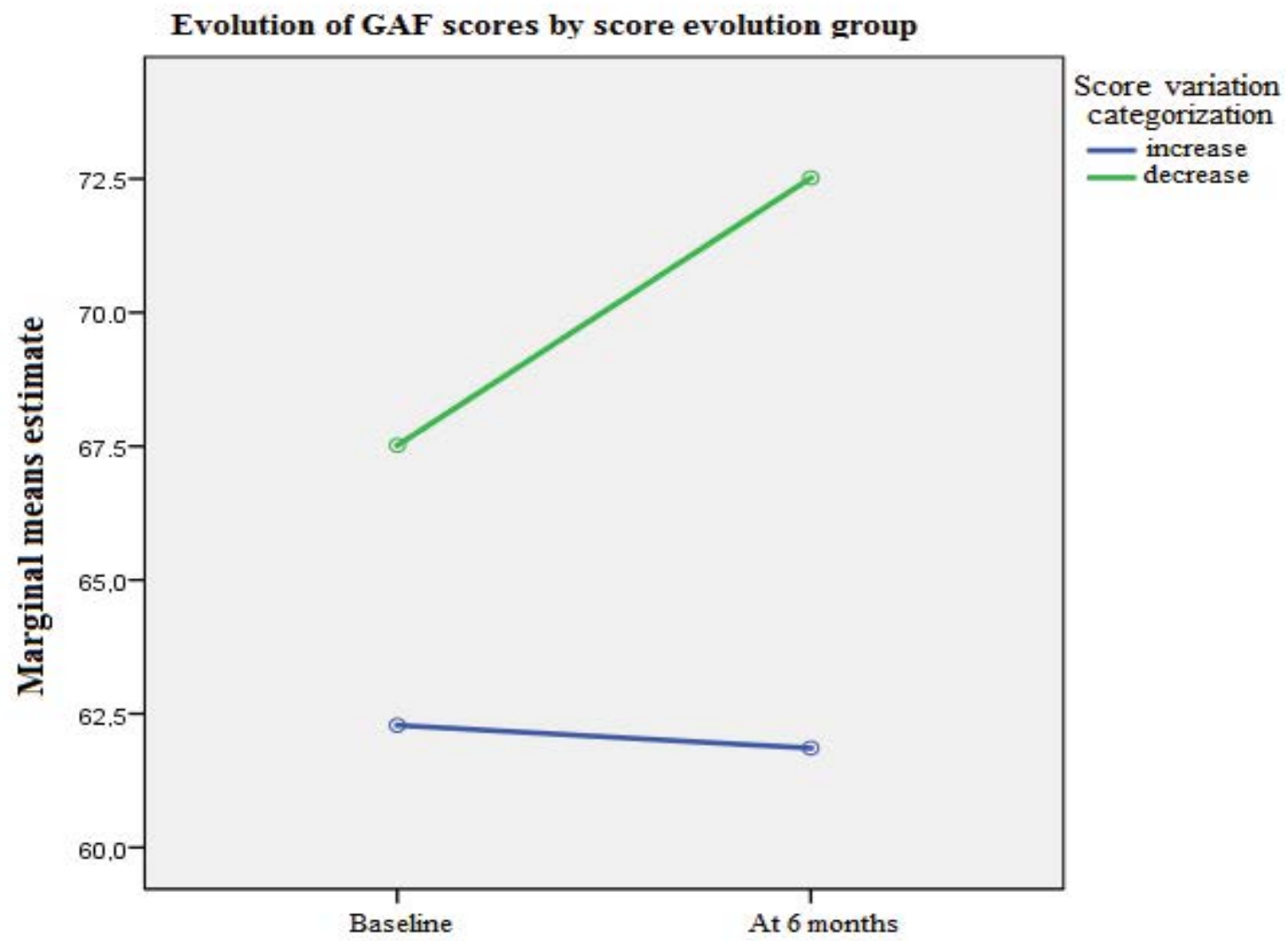

Figure 2: Evolution of functioning with respect to shifts in the BDNF receptor FL/T ratio.

Thus, in patients with a low to medium FL/T ratio, a higher educational level was significantly associated with a better baseline working memory and IQ (Table 2).

In light of the interaction observed between baseline $\mathrm{FL} / \mathrm{T}$ and educational level, we investigated the impact of educational level on the cognitive function of FEP patients as a post-hoc result. After adjusting for IQ, we observed that completion of secondary or higher education studies was significantly associated with a faster speed of processing $(\beta=0.483$, $p=0.030$ and $\beta=0.592, p=0.034)$. Furthermore, higher education was correlated with a better working memory $(\beta=0.619, p=0.008)$.
As it occurred with baseline cognition, a higher FL/T ratio significantly correlated with a better overall cognitive function $(\beta=70.769, p=0.003)$ and verbal memory $(\beta=0.830, p=0.011)$ two years after the FEP.

No significant correlation was found between shifts in the FL/T ratio (as continuous variable) and cognitive performance. Nevertheless, when TrKB-FL/ TrKB-T ratio was higher than> 1 (as a qualitative variable when BDNF fulllength receptor levels exceed those of truncated receptors), cognition was better both at baseline (IQ: $\beta=10.266, p=0.002$; Processing speed: $\beta=0.502, p=0.029$; CGI: $\beta=18.339, p=0.006$ ) and at two years (Verbal memory: $\beta=0.620$, 


Table 2: Effect size of baseline FL/T on baseline cognitive function by educational level.
\begin{tabular}{|l|ll|ll|}
\hline & \multicolumn{3}{l|}{ Baseline IQ } & Baseline working memory \\
\hline $\begin{array}{l}\text { Percentiles (FL/T ratio } \\
\text { value) }\end{array}$ & $\begin{array}{l}\text { Medium level } \\
\text { Effect size (CI) }\end{array}$ & $\begin{array}{l}\text { High Level } \\
\text { Effect size (CI) }\end{array}$ & $\begin{array}{l}\text { Medium level } \\
\text { Effect size (CI) }\end{array}$ & $\begin{array}{l}\text { High Level } \\
\text { Effect size (CI) }\end{array}$ \\
\hline P3 (0.18) & $27.87(10.75,45)$ & $41.38(22.73,60.03)$ & $0.70(-0.254,1.654)$ & $1.84(0.814,2.866)$ \\
P25 (0.67) & $13.27(4.32,22.22)$ & $23.72(13.73,33.71)$ & $0.38(-0.156,0.916)$ & $1.20(0.606,1.794)$ \\
P50 (0.90) & $6.41(-1.19,14.02)$ & $15.43(6.65,24.21)$ & $0.20(-0.261,0.661)$ & $0.85(0.316,1.384)$ \\
P75 (1.11) & $0.15(-8.84,9.14)$ & $7.86(-2.56,18.28)$ & $0.04(-0.516,0.596)$ & $0.53(-0.117,1.177)$ \\
P97 (1.96) & $-25.18(-49.62,-0.74)$ & $-22.78(-50.08,4.52)$ & $-0.60(-2.106,0.906)$ & $-0.75(-2.426,0.926)$ \\
\hline
\end{tabular}

$\mathrm{p}=0.020$; CGI: $\beta=28.488, p=0.004)$. This effect can be due both to an increase of TrKB-FL or a decrease of TrKB-T, or both.

\section{Discussion}

Appropriate levels of neurotrophins -and their specific receptors- are required for adequate neuronal response and survival. The results of this study reveal that cognitive performance improves when BDNF full-length receptor levels exceed those of truncated receptors. This finding supports our hypothesis that the role of neurotrophin receptors is more relevant than that of neurotrophins themselves. Neurotrophin receptors help neurotrophins modulate brain plasticity, which is related to cognitive performance. The increase of this index (FL/T ratio) in the first six months of treatment after a first psychotic episode is also associated with better functioning. When the FL/T ratio increases over time global functioning improves. Yet, to improve the cognitive performance of the patient, it is necessary that the expression of the functional receptor is greater than the expression of the truncated receptor. This may be due to the fact that cognitive processes generate free radicals, which requires a more effective repair system.

Increased TrkB-FL levels in the long term with respect to the truncated form may be an adaptive response to cellular damage at disease onset. In a previous study, we observed that BDNF receptor expression at disease onset is related to patient's response to treatment [6]. The reason why $\mathrm{BDNF}$ is not a reliable biomarker for cognition may be that it dilutes in plasma. Also, there is evidence that the role of BDNF as a marker of response to cognitive remediation in patients with schizophrenia depends on polymorphisms of the BDNF gene [19].

Inconsistent data have been published on peripheral BDNF levels in patients with schizophrenia $[7,20,21]$ or a FEP[22,23].

Our results can be explained by several mechanisms. The truncated form of the $\operatorname{TrKB}$
(TrKB-T) receptor sequesters BDNF, competes with TrKB-FL, and uses a different signaling pathway [11]. In an in vitro study, Dorsey et al (2012) observed that synaptic function improves in the absence of TrKB-T [24]. This means that increased TrKB-T receptor levels impair synaptic function and inhibit the neurotrophic function of BDNF [25]. Indeed, BDNF overexpression prevents cognitive deterioration in adolescent cannabis users [26]. Moreover, BDNF-mediated cellular survival has been reported to decrease when TrKB-T is overexpressed in an animal model of Down's syndrome [27].

Our study revealed that a higher educational level is related to better cognitive performance after a FEP [28]. Other studies in elderly population have documented that a high education level has as protective effect against cognitive impairment [29]. Additionally, higher education has been proven to be a predictor of global functioning and quality of life in patients with schizophrenia [30].

Negative results have been obtained in intervention studies where BDNF was administered to animals. These results may be due to difficulties in the administration of BDNF to animals. Also, its pharmacokinetics is unknown [31], as neurotrophins are large proteins with a poor penetration into tissue that cannot enter the blood-brain barrier. $\operatorname{TrKB}$ receptor activation is known to have neuroprotective effects [32]. Furthermore, there is evidence that $\operatorname{TrKB}$ receptors can be activated using low-molecular-weight molecules such as adenoside/adenosine agonists in neuronal populations with $\mathrm{TrkB}$ and adenosine receptor expression [33]. Glucocorticoids have also been shown to promote $\mathrm{TrKB}$ activation -especially in neurogenic CNS regions-, with no increase in BDNF expression in vitro and in vivo in animals [34]. Studies are warranted in the future to determine whether the $\mathrm{TrkB}$ receptor that activates in the absence of BDNF is TrKB-FL or TrKB-T.

Dorsey et al reversed neuronal death by restoring TrKB-FL expression in experimental animals 
[35]. Thus, TrKB-FL could be the mediating factor that explains that overall and cognitive functioning improves when TrKB-FL and TrKB-T ratio is adequate. Levels of BDNF receptor isoforms in areas related to cognition such as the hippocampus- have been associated with cognitive decline in aged rats [36]. According to Dorsey, TrKB-T overexpression with respect to TrKB-FL is a disruptor of the BDNF signaling pathway.

Under normal conditions, TrKB-T expresses in the presence of exogenous BDNF to metabolize FL-BDNF complexes and regulate TrKB-FL activity [37]. In patients with schizophrenia, the BDNF signaling pathway may be altered from the first psychotic episode. According to Dorsey, TrKB-T overexpression with respect to TrKB-FL is a disruptor of the BDNF signaling pathway. Further studies based on the synthesis of these receptors (mRNA, PCR) are needed to determine changes in FL/T receptor expression.

A limitation of the study concerns evidence that BDNF serum levels and TrK-B receptors are related to traumatic experiences [38,39] and this factor has not been taken into account in this research. Furthermore, we have focused on one of the receptors of BDNF. The other receptor, $\mathrm{p} 75$, has not been measured in this study. Another limitation is that although the truncated form is considered nonfunctional in CNS, it inhibits p75 mediated neuronal death induced by BDNF.

The strengths of the study include that a highly homogeneous sample in terms of age, duration of illness, and pharmacological treatment. Strength is long-term follow-up.

In conclusion, levels of BDNF receptors during first episode psychosis and at two years are related to cognitive and overall functioning. Further research is warranted to explore if cognitive and functional deterioration in patients with psychosis can be prevented by an intervention on the BDNF signaling pathway.

\section{Conflict of Interests}

The authors declare no conflicts of interest.

\section{Acknowledgements}

This work was supported by the Spanish Ministry of Economy and Competiveness and the Carlos III Health Institute through CIBERSAM, and the Foundation Santander-UCM (GR 58/08), and partly supported by the Spanish Ministry of Economy and Competiveness directly, and by the European Regional Development Fund, FEDER (PI10/01746, PI11/01977, PI11/02708, PI12/02077, PI14/01900, PI15/00299, PI15/00789, PI16/01164), local grants from the Department of Education, Linguistic Policy and Culture of the Basque Country Government (2010111170, 2010112009), the Basque Foundation for Health Innovation and Research (BIOEF), ) and the University of the Basque Country (GIC10/80, GIC12/84 (IT679-13))

We want to particularly acknowledge the patients enrolled in this study for their participation and the Basque Biobank for its collaboration

\section{References}

1. Parellada M, Gomez-Vallejo S, Burdeus $M$ et al. Developmental Differences between Schizophrenia and Bipolar Disorder. Schizophr. Bull 43(6), 1176-1189 (2017).

2. Palomino $A$, Vallejo-lllarramendi $A$, González-Pinto A, et al. Decreased levels of plasma BDNF in first-episode schizophrenia and bipolar disorder patients. Schizophr. Res 86(1-3), 321-322 (2006).

3. Fernandes BS, Steiner J, Berk M, et al. Peripheral brain-derived neurotrophic factor in schizophrenia and the role of antipsychotics: meta-analysis and implications. Mol. Psychiatry 20(9), 1108-1119 (2015).

4. González-Pinto A, Mosquera F, Palomino A, et al. Increase in brain-derived neurotrophic factor in first episode psychotic patients after treatment with atypical antipsychotics. Int. Clin. Psychopharmacol 25(4), 241-245
(2010).

5. Ruiz de Azua S, Matute C, Stertz L, et al. Plasma brain-derived neurotrophic factor levels, learning capacity and cognition in patients with first episode psychosis. BMC Psychiatry 13, 27 (2013).

6. Martinez-Cengotitabengoa $M, M a c D o w e l l$ KS, Alberich S, et al. BDNF and NGF Signalling in Early Phases of Psychosis: Relationship With Inflammation and Response to Antipsychotics After 1 Year. Schizophr. Bull 42(1), 142-151 (2016).

7. Green MJ, Matheson SL, Shepherd A, et al. Brain-derived neurotrophic factor levels in schizophrenia: a systematic review with meta-analysis. Mol. Psychiatry 16(9), 960972 (2011).

8. Heitz U, Papmeyer M, Studerus E, et al. Plasma and serum brain-derived neurotrophic factor (BDNF) levels and their association with neurocognition in at-risk mental state, first episode psychosis and chronic schizophrenia patients. World J. Biol. Psychiatry Off. J. World Fed. Soc. Biol. Psychiatry 1-10 (2018).

9. Ahmed AO, Mantini AM, Fridberg DJ, et al. Brain-derived neurotrophic factor (BDNF) and neurocognitive deficits in people with schizophrenia: a meta-analysis. Psychiatry Res 226(1), 1-13 (2015).

10. Man L, Lv X, Du X-D, et al. Cognitive impairments and low BDNF serum levels in first-episode drug-naive patients with schizophrenia. Psychiatry Res 263, 1-6 (2018).

11. Fenner BM. Truncated TrkB: beyond a dominant negative receptor. Cytokine Growth Factor Rev 23(1-2), 15-24 (2012).

12. Yang $Y-J, L i Y-K$, Wang $W$, et al. Small-molecule TrkB agonist 7, 8-dihydroxyflavone reverses cognitive and synaptic plasticity 


\section{Research Mónica Martínez-Cengotitabengoa}

deficits in a rat model of schizophrenia. Pharmacol. Biochem. Behav 122, 30-36 (2014).

13. Devi L, Ohno M. 7, 8-dihydroxyflavone, a small-molecule TrkB agonist, reverses memory deficits and BACE1 elevation in a mouse model of Alzheimer's disease. Neuropsychopharmacol. Off. Publ. Am. Coll. Neuropsychopharmacol 37(2), 434-444 (2012).

14. García-Bueno B, Bioque M, Mac-Dowell KS, et al. Pro-/anti-inflammatory dysregulation in patients with first episode of psychosis: toward an integrative inflammatory hypothesis of schizophrenia. Schizophr. Bull 40(2), 376-387 (2014)

15. Kay SR, Fiszbein A, Opler LA. The positive and negative syndrome scale (PANSS) for schizophrenia. Schizophr. Bull 13(2), 261-276 (1987).

16. Klein AB, Williamson $R$, Santini MA, et al. Blood BDNF concentrations reflect brain-tissue BDNF levels across species. Int. J. Neuropsychopharmacol 14(3), 347-353 (2011).

17. Pillai A, Kale A, Joshi S, et al. Decreased BDNF levels in CSF of drug-naive first-episode psychotic subjects: correlation with plasma BDNF and psychopathology. Int. J. Neuropsychopharmacol 13(4), 535-539 (2010).

18. Endicott J, Spitzer RL, Fleiss JL, et al. The global assessment scale. A procedure for measuring overall severity of psychiatric disturbance. Arch. Gen. Psychiatry 33(6), 766-771 (1976).

19. Penadés R, López-Vílchez I, Catalán R, et al. BDNF as a marker of response to cognitive remediation in patients with schizophrenia: A randomized and controlled trial. Schizophr. Res (2017).

20. Martinotti G, Di lorio G, Marini S, et al. Nerve growth factor and brain-derived neurotrophic factor concentrations in schizophrenia: a review. J. Biol. Regul. Homeost. Agents 26(3), 347-356 (2012)

21. Libman-Sokołowska M, Drozdowicz $E_{\text {, }}$ Nasierowski T. BDNF as a biomarker in the course and treatment of schizophrenia. Psychiatr. Pol 49(6), 1149-1158 (2015).

22. Goto N, Yoshimura R, Kakeda S, et al. Comparison of brain $\mathrm{N}$-acetylaspartate levels and serum brain-derived neurotrophic factor (BDNF) levels between patients with first-episode schizophrenia psychosis and healthy controls. Eur. Psychiatry J. Assoc. Eur. Psychiatr 26(1), 57-63 (2011).

23. Toll $A$, Mané $A$. Brain-derived neurotrophic factor levels in first episode of psychosis: $A$ systematic review. World J. Psychiatry 5(1), 154-159 (2015)

24. Dorsey SG, Lovering RM, Renn CL, et al. Genetic deletion of trkB.T1 increases neuromuscular function. Am. J. Physiol. Cell Physio 302(1), C141-153 (2012).

25. Eide FF, Vining ER, Eide BL, et al. Naturally occurring truncated trkB receptors have dominant inhibitory effects on brain-derived neurotrophic factor signaling. $J$. Neurosci. Off. J. Soc. Neurosci 16(10), 31233129 (1996).

26. Segal-Gavish H, Gazit N, Barhum Y, et al. BDNF overexpression prevents cognitive deficit elicited by adolescent cannabis exposure and host susceptibility interaction. Hum. Mol. Genet 26(13), 2462-2471 (2017).

27. Dorsey SG, Bambrick LL, Balice-Gordon RJ, et al. Failure of brain-derived neurotrophic factor-dependent neuron survival in mouse trisomy 16. J. Neurosci. Off. J. Soc. Neurosci 22(7), 2571-2578 (2002)

28. Amoretti S, Bernardo M, Bonnin CM, et al. The impact of cognitive reserve in the outcome of first-episode psychoses: 2-year follow-up study. Eur. Neuropsychopharmacol. J. Eur. Coll. Neuropsychopharmacol 26(10), 1638-1648 (2016).

29. Lee W-J, Liang C-K, Peng L-N, et al. Protective factors against cognitive decline among community-dwelling middle-aged and older people in Taiwan: A 6-year national population-based study. Geriatr. Gerontol. Int 17 Suppl 1, 20-27 (2017).

30. Siegel SJ, Irani F, Brensinger CM, et al. Prognostic variables at intake and long-term level of function in schizophrenia. Am. J. Psychiatry 163(3), 433-441 (2006).

31. Thoenen $\mathrm{H}$, Sendtner M. Neurotrophins: from enthusiastic expectations through sobering experiences to rational therapeutic approaches. Nat. Neurosci 5 Suppl, 1046-1050 (2002)

32. Rajagopal R, Chen Z-Y, Lee FS, et al. Transactivation of Trk neurotrophin receptors by G-protein-coupled receptor ligands occurs on intracellular membranes. J. Neurosci. Off. J. Soc. Neurosci 24(30), 6650-6658 (2004).

33. Domeniconi M, Chao MV. Transactivation of Trk receptors in spinal motor neurons. Histol. Histopathol 25(9), 1207-1213 (2010).

34. Jeanneteau F, Garabedian MJ, Chao MV. Activation of Trk neurotrophin receptors by glucocorticoids provides a neuroprotective effect. Proc. Natl. Acad. Sci. U. S. A. 105(12), 4862-4867 (2008).

35. Dorsey SG, Renn CL, Carim-Todd L, et al. In vivo restoration of physiological levels of truncated TrkB.T1 receptor rescues neuronal cell death in a trisomic mouse model. Neuron 51(1), 21-28 (2006).

36. Silhol M, Bonnichon V, Rage F, et al. Age-related changes in brain-derived neurotrophic factor and tyrosine kinase receptor isoforms in the hippocampus and hypothalamus in male rats. Neuroscience 132(3), 613-624 (2005).

37. Wong J, Garner B. Evidence that truncated TrkB isoform, TrkB-Shc can regulate phosphorylated TrkB protein levels. Biochem. Biophys. Res. Commun 420(2), 331-335 (2012).

38. Angelucci F, Ricci V, Gelfo F, et al. BDNF serum levels in subjects developing or not post-traumatic stress disorder after trauma exposure. Brain Cogn 84(1), 118-122 (2014).

39. Andero R, Choi DC, Ressler KJ. BDNF-TrkB receptor regulation of distributed adult neural plasticity, memory formation, and psychiatric disorders. Prog. Mol. Biol. Transl. Sci 122, 169-192 (2014). 